\title{
Meromorphic Non-Integrability of Several 3D Dynamical Systems
}

\author{
Kaiyin Huang ${ }^{1,2}$, Shaoyun Shi ${ }^{1,2}$ and Wenlei $\mathrm{Li}^{1,3, *}$ \\ 1 School of Mathematics, Jilin University, Changchun 130012, China; keiyinhuang@gmail.com (H.K.); \\ shisy@jlu.edu.cn (S.S.) \\ 2 State Key Laboratory of Automotive Simulation and Control, Jilin University, Changchun 130012, China \\ 3 Beijing Computational Science Research Center, Haidian District, Beijing 100094, China \\ * Correspondence: lwlei@jlu.edu.cn; Tel.: +86-431-85166042
}

Academic Editor: Gunnar Pruessner

Received: 4 March 2017; Accepted: 29 April 2017; Published: 10 May 2017

\begin{abstract}
In this paper, we apply the differential Galoisian approach to investigate the meromorphic non-integrability of a class of 3D equations in mathematical physics, including Nosé-Hoover equations, the Lü system, the Rikitake-like system and Rucklidge equations, which are well known in the fields of molecular dynamics, chaotic theory and fluid mechanics, respectively. Our main results show that all these considered systems are, in fact, non-integrable in nearly all parameters.
\end{abstract}

Keywords: differential Galois theory; first integrals; meromorphic non-integrability

\section{Introduction}

One important problem in the qualitative theory of nonlinear differential dynamic systems is understanding its complexity and chaotic properties. Integrability can be regarded as a good breakthrough. Roughly speaking, a system is integrable if it possesses such a number of first integrals that it is solvable by quadratures. Using a first integral of the considered system, we can reduce the dimension of that by one. Enough functionally independent first integrals can help us get general solutions analytically in an "explicit" way and understand the topological structure. The non-existence of first integrals can allow us to expect that the system is complex and admits chaotic behavior.

We note that non-integrability of systems is a first step towards proving that this system is chaotic. To deal with the issue, one should show that the system has positive Lyapunov exponents or metric entropy, heteroclinic connections, and so on (see for instance [1-3]). There is much literature on the complex behavior and non-integrability of dynamical systems. For example, Bolsinov and Taimanov [4] constructed a geodesic flow on a real-analytic Riemannian manifold $M_{A}$ which is integrable with $C^{\infty}$ first integrals but has positive topological entropy. Yagasaki [5] obtained a equivalent condition between the existence of a simple zero for the Melnikov function of a class of two degree-of-freedom Hamiltonian systems with saddle centers and the non-commutativity of the differential Galois group of corresponding normal variational equations.

There is no general approach to detect the existence of first integrals for the considered differential system. Many scholars have devoted themselves to this topic and developed a lot of ways to study the existence of first integrals for given systems, such as the Lax pairs [6], the Painlevé analysis [7], the Lie symmetries [8] and the Darboux integrability theory [9]. Inspired by Ziglin's works [10], Morales-Ruiz et al. [11-16] applied the differential Galois theory to the non-integrability of Hamiltonian systems with great success. Roughly speaking, the Morales-Ramis theory shows that if the Hamiltonian system with $n$ degrees of freedom admits $n$ meromorphic first integrals which are in involution and independent, then the identity component of the differential Galois group of the normal variational equation should be commutative. Since then, Morales-Ramis theory has been considered as a powerful 
tool for the meromorphic non-integrability of the Hamiltonian system, and has been successfully applied by many scholars to large numbers of physical models, such as by the authors of [17-20].

For non-Hamiltonian system, the authors [21,22] proposed two analogous forms of the Morales-Ramis theory for general dynamical systems both in the form of vector fields and mappings, which can be, in fact, viewed as a natural generalization of the Ziglin theorem and Morales-Ramis theory to non-Hamiltonian systems. The main idea behind these results is that the number of functionally independent meromorphic first integrals of given differential equations implies the structure of the identity component of the differential Galois group of the normal variational equations along some non-equilibrium solution. In addition, there are some other results on the Galoisian obstruction to the integrability of non-Hamiltonian systems (see $[23,24]$ ).

It is very interesting to note that, for 3D differential systems, there are a large number of applications of the non-existence of first integrals in some given function spaces such as polynomial, analytic, rational, and algebraic first integral applications, etc. However, to our knowledge, there are few applications for the non-existence of meromorphic first integrals of 3D differential systems (see the Arnold-Beltrami-Childress(ABC) flow [25,26] and the steady Stokes flow [27]).

In this work, given a three-dimensional differential system depending on some parameters, we will present a systematic approach to recognize the values of parameters for which equations have meromorphic first integrals or not. Then we will apply our results to several 3D differential systems, including Nosé-Hoover equations [28], the Rikitake-like system [29], Lü system [30] and Rucklidge equations [31]. Moreover, the tools we use to study the meromorphic non-integrability can be applied to other three-dimensional differential systems.

This paper is organized as follows. In Section 2, we present some preliminary results that will be used later and give a systematic approach to study the non-existence of meromorphic first integrals for three-dimensional differential systems. In Section 3, we study several three-dimensional systems and obtain some results about the meromorphic non-integrability of them.

\section{Preliminaries}

Consider the following $n$-dimensional analytic differential system:

$$
\dot{X}=F(X), \quad X \in \mathcal{M},
$$

where the dot is the derivation with respect to $t \in \mathbb{C}, \mathcal{M}$ is a $n$-dimensional complex analytic manifold and $F$ is a vector-valued analytic function. Let $U$ be an open set in the manifold $\mathcal{M}$. A non-constant function $\Phi: U \rightarrow \mathbb{C}$ is called a first integral of (1) if it is constant along any solution curve of system (1). If system (1) admits $n-1$ functionally independent first integrals $F_{1}(x), \cdots, F_{n-1}(x)$, then we say that system (1) is completely integrable, which can help us to get the global dynamical information of (1). In this case, the orbits of this system are contained in the curves:

$$
\left\{x \mid F_{1}(x)=c_{1}, \cdots, F_{n-1}(x)=c_{n-1}\right\},
$$

where $c_{1}, \cdots, c_{n-1}$ are constants, and its general solutions can be obtained analytically in an "explicit" way. Moreover, the topological structure of a completely integrable system is also simple. Indeed, if system (1) admits $n-1$ functionally independent first integrals of class $C^{r}$ with $r>2$, then it is $C^{r-1}$ orbitally equivalent to a linear differential system, for more details see [32].

If system (1) admits $0<m<n-1$ first integrals, i.e., "partially integrable", then we can replace the considered system with an $n-m$-dimension reduced one. The non-existence of first integrals for (1) may always push people to investigate the complex phenomena. 
Let $\varphi(t)$ be a non-equilibrium analytic solution of system (1). Denote by $\Gamma$ the Riemann surface corresponding to this particular solution $\varphi(t)$. The variational equations along $\Gamma$ are given by:

$$
\dot{\xi}=A(t) \xi, A(t)=\left.\frac{\partial F}{\partial X}\right|_{X=\varphi(t)}, \xi \in T_{\Gamma} \mathcal{M},
$$

where $T_{\Gamma}$ is the vector bundle of $T \mathcal{M}$ restricted on $\Gamma$. Let the mapping $\pi$ be the nature projection from the $T_{\Gamma}$ to the normal bundle $T_{\Gamma} / T \Gamma$. Then, the variational Equation (2) can be reduced to the normal variational equations:

$$
\dot{\eta}=\pi_{*}\left(T F\left(\pi^{-1} \eta\right)\right), \quad \eta \in T_{\Gamma} / T \Gamma .
$$

Since (3) is a linear differential equation, we can consider the differential Galois group corresponding to (3). Generally speaking, the differential Galois group $G$ of (3) is a matrix subgroup of $G L(n-1, \mathbb{C})$ acting on the fundamental solutions of (3) such that it dose not change polynomial and differential relations between them. To be more precise, the differential Galois group $G$ of (3) is the group of all differential automorphisms of the field $K\left(\eta_{1}(t), \cdots, \eta_{n-1}(t)\right)$ such that any element of the field $K$ is fixed, where $K$ is the differential field consisting of all meromorphic functions over $T_{\Gamma} / T \Gamma$ and $\left(\eta_{1}(t), \cdots, \eta_{n-1}(t)\right)$ is a fundamental solution matrix of (3). For any $\sigma \in G$, there exists a non-singular $(n-1) \times(n-1)$ matrix $M_{\sigma}$ such that:

$$
\left(\sigma\left(\eta_{1}\right), \cdots, \sigma\left(\eta_{n-1}\right)\right)=\left(\eta_{1}, \cdots, \eta_{n-1}\right) M_{\sigma} .
$$

Therefore, $G$ is isomorphic to a subgroup of $G L(n-1, \mathbb{C})$. Moreover, it can be shown that $G$ is a linear algebraic group [33] and has a unique connected component $G^{0}$ containing the identity which is called the identity component of $G$. For basic notions and results of the differential Galois theory, one can consult the book [33].

Now we state our results in $[21,22]$ which reveal that the number of meromorphic first integrals of given systems is reflected by the properties of the identity component $G^{0}$.

Theorem 1. Assume that system (1) has $m(1 \leq m<n)$ functionally independent meromorphic first integrals in a neighborhood of $\Gamma$. Then, the Lie algebra $\mathcal{G}$ of the differential Galois group $G$ of Equation (3) has $m$ meromorphic invariants, and the identity component $G^{0}$ of $G$ has at most $(n-m-1)(n-1)$ generators, i.e.,

$$
G^{0}=\left\{\left(e^{\mathfrak{T}_{1} t_{1}} \cdot e^{\mathfrak{T}_{2} t_{2}} \cdots e^{\mathfrak{T}_{k} t_{k}}\right)^{s} \mid\left(t_{1}, \cdots, t_{k}\right) \in \tilde{\mathcal{V}} \subset \mathbb{C}^{k}, s \in \mathbb{N}\right\},
$$

where $\left\{\mathfrak{T}_{1}, \cdots, \mathfrak{T}_{k}\right\}$ is a basis of $\mathcal{G}$ with $k \leq(n-m-1)(n-1), \tilde{V}$ is a neighborhood of the original element in $\mathbb{C}^{l}$. In particular,

(1) If $m=n-1$, i.e., system (1) is completely integrable, then $\mathcal{G}=\{0\}, G^{0}=\{\mathbf{1}\}$, where $\mathbf{1}$ denotes the identity element of $G$.

(2) If $m=n-2$, then $\mathcal{G}, G^{0}$ has at most $n-1$ generators.

(3) If $n=3$ and $m=1$, then $\mathcal{G}, G^{0}$ are solvable.

As the identity component $G^{0}$ is a normal subgroup of $G$ with finite index, $G^{0}$ is the trivial subgroup $\{\mathbf{1}\}$ if and only if $G$ is a finite group. Then, we have the following simple conclusion which will be used in this paper.

Corollary 1. Let $n=3$. If the differential Galois group of (3) is not finite, then system (1) is not completely integrable with meromorphic first integrals. Further, if the identity component $G^{0}$ of (3) is not solvable, then system (1) has no any meromorphic first integral. 
Based on the above results, one can easily conclude an unified approach to analyze the meromorphic non-integrability in certain 3-dimensional nonlinear dynamical systems by means of the differential Galois theory as follows.

Step 1. Find a non-equilibrium solution for the considered system.

Step 2. Get the normal variational equations along the obtained particular solution.

Step 3. Compute, or analyze the differential Galois group of the normal variational equations.

We remark that, to carry out above systematic steps, there are two key points one should pay attention to. Firstly, in order to derive the normal variational equations easily, we should select a proper particular solution of the considered differential equations. In many cases, the non-equilibrium particular solution $\varphi(t)$ of (1) can be a straight line solution. Secondly, there is no general approach to calculate the differential Galois group of a linear differential equation. In some particular cases, one can compute the differential Galois group by the property of the monodromy group or the solvability of second-order linear differential equations, see for instance $[17,18]$. For second-order linear differential equations with rational coefficients, the so-called Kovacic's algorithm [34] is a very effective tool to calculate the differential Galois group. In addition, we can also transform the normal variational equations into some famous equations such as the Riemann P equation, Bessel equation and Whittaker equation, for which their differential Galois group is well-known. See Appendixes A and B for more details.

\section{Non-Integrability}

In what follows, we will apply the systematic Galoisian approach concluded in the previous section to investigate the meromorphic non-integrability of several 3D systems, including Noseé-Hoover equations, the Lü system, the Rikitake-like system and Rucklidge equations, which are well known in the fields of molecular dynamics, chaotic theory and fluid mechanics, respectively.

\subsection{Nosé-Hoover System}

Consider the Nosé-Hoover equations for one-dimensional harmonic oscillator:

$$
\left\{\begin{array}{l}
\dot{x}=-y-x z \\
\dot{y}=x \\
\dot{z}=\alpha\left(x^{2}-1\right),
\end{array}\right.
$$

where $x$ represents momentum, $y$ represents the oscillator coordinate and $z$ represents the friction coefficient [28]. This model describes the interaction of a particle with a heat-bath. The Nosé-Hoover system plays an important role in molecular dynamics and can be used to compute phase space integrals for the canonical Gibbs distribution. From the dynamical point of view, it was intensively studied in [35-37].

When the parameter $\alpha$ vanishes, system (4) admits a first integral $F_{1}=z$, and can be reduced into a linear system when it is restricted to the level set of $F_{1}$. Hence, in this case, we can get its general solutions analytically in an "explicit" way. Therefore, from the view of integrability, system (4) with $\alpha=0$ is trivial, and we need only deal with system (4) with $\alpha \neq 0$. Moreover, as pointed out by Mahdi and Valls [37], system (4) can also be viewed as a completely integrable system with the two functionally independent first integrals.

Assume $\alpha \neq 0$. Then, it is easy to check that a non-equilibrium solution to (4) is expressed as $(x, y, z)=(0,0,-\alpha t)$. Let $\Gamma_{1}$ be the phase curve associated with this particular solution. Taking 
$(x, y, z)=(\xi, \eta,-\alpha t+\zeta)$ in (4) and neglecting quadratic terms of $\xi, \eta, \zeta$, we obtain the variational equations along $\Gamma_{1}$,

$$
\left(\begin{array}{l}
\dot{\zeta} \\
\dot{\eta} \\
\dot{\zeta}
\end{array}\right)=\left(\begin{array}{ccc}
-\alpha t & -1 & 0 \\
1 & 0 & 0 \\
0 & 0 & 0
\end{array}\right)\left(\begin{array}{l}
\xi \\
\eta \\
\zeta
\end{array}\right),
$$

the corresponding normal variational equations are:

$$
\left(\begin{array}{l}
\dot{\xi} \\
\dot{\eta}
\end{array}\right)=\left(\begin{array}{cc}
-\alpha t & -1 \\
1 & 0
\end{array}\right)\left(\begin{array}{l}
\xi \\
\eta
\end{array}\right) .
$$

Eliminating $\eta$ leads to:

$$
\ddot{\xi}+\alpha t \dot{\xi}+(1+\alpha) \xi=0 .
$$

Making the change of variable $\xi(t)=\chi(t) \exp \left(-\alpha t^{2} / 4\right)$, we have:

$$
\ddot{\chi}=r(t) \chi, r(t)=\left(\frac{\alpha^{2} t^{2}}{4}-\frac{\alpha+2}{2}\right) .
$$

Since (7) has no pole and the order at $\infty$ is $0-2=-2$, by Theorem A2, case 3 in Theorem A1 cannot occur. Hence, the differential Galois group of (7) is not finite, namely, the identity component is not the trivial subgroup, which implies the differential Galois group of (5) is also not finite. Therefore, system (4) is not completely integrable with meromorphic first integrals by Corollary 1 . Further, in view of Theorem A4, we have:

$$
a=\frac{\alpha}{2}, b=0, c=-\frac{\alpha+2}{2},
$$

and,

$$
\frac{4 a c-b^{2}}{4 a^{2}}=-\frac{\alpha+2}{\alpha} .
$$

Hence, the identity component of the differential Galois group of (7) is solvable if and only if $-(\alpha+2) / \alpha$ is an odd integer, i.e., $1 / \alpha \in \mathbb{Z}$.

Thanks to Corollary 1 , we have the following results.

Theorem 2. Nosé-Hoover Equation (4) is not meromorphic completely integrable when $\alpha \neq 0$. Moreover, if $n \alpha \neq 1$ for any $n \in \mathbb{Z}$, then Nosé-Hoover Equation (4) does not admit any meromorphic first integral in a neighbourhood of $\Gamma_{1}$.

When $n \alpha=1$ for some integer $n$, the above result does not tell whether Nosé-Hoover Equation (4) admits meromorphic first integrals or not. However, if we can find another particular solution of (4), then the remaining values may be removed. Similar techniques have been applied to the non-integrability of the generalized spring-pendulum Hamiltonian system [19]. Indeed, assume $\alpha \neq 1$, then (4) has another straight-line solution $x=k, y+k z=0$, i.e.,

$$
(x(t), y(t), z(t))=(k, k t,-t),
$$

where $k^{2}=1-1 / \alpha$. Let $\Gamma_{1}^{\prime}$ be the phase curve associated with this particular solution.

Theorem 3. Assume $n \alpha=1$ for certain $n \in \mathbb{Z}$ and $\alpha \notin\{1,1 / 2,-1 / 2\}$. Then Nosé-Hoover Equation (4) does not admit any meromorphic first integral in a neighbourhood of $\Gamma_{1}^{\prime}$. 
Proof. Proceeding as above, the variational equations along $\Gamma_{1}^{\prime}$ reads:

$$
\left(\begin{array}{c}
\dot{\zeta} \\
\dot{\eta} \\
\dot{\zeta}
\end{array}\right)=\left(\begin{array}{ccc}
t & -1 & -k \\
1 & 0 & 0 \\
2 \alpha k & 0 & 0
\end{array}\right)\left(\begin{array}{l}
\xi \\
\eta \\
\zeta
\end{array}\right) .
$$

Since we have $x=k, y+k z=0$ along the phase curve $\Gamma_{1}^{\prime}$, then $\xi_{1}=\xi$ and $\eta_{1}=\eta+k \zeta$ can be taken as coordinates in which the normal variational equations are given by:

$$
\left(\begin{array}{l}
\dot{\xi}_{1} \\
\dot{\eta}_{1}
\end{array}\right)=\left(\begin{array}{cc}
t & -1 \\
2 \alpha-1 & 0
\end{array}\right)\left(\begin{array}{l}
\xi_{1} \\
\eta_{1}
\end{array}\right),
$$

or,

$$
\ddot{\xi}_{1}=t \dot{\xi}_{1}+(2-2 \alpha) \xi_{1}
$$

Making the change of variable $\xi_{1}(t)=\chi_{1}(t) \exp \left(t^{2} / 4\right)$ yields:

$$
\ddot{\chi}_{1}=r(t) \chi_{1}, r(t)=\frac{t^{2}}{4}+\frac{3-4 \alpha}{2} .
$$

We claim that the differential Galois group of (9) is not solvable. Indeed, if the differential Galois group of (9) is solvable, then it follows from Theorem A4 that $3-4 \alpha=3-4 / n$ is an odd integer, which contradicts the assumption.

Therefore, the differential Galois group of (9) is not solvable. By Corollary 1, the conclusion is proved.

Remark 1. When $\alpha=1 / 2$ or $\alpha=-1 / 2$, (9) admits a solution:

$$
\chi_{1}(t)=\exp \left(t^{2} / 4\right) \text { or } \chi_{1}(t)=\exp \left(t^{2} / 4\right)\left(t^{2}+1\right),
$$

and the differential Galois group of (9) is solvable, and we can not get the information on the integrability of (4) from Corollary 1.

In view of the above results, we have the following corollary on the meromorphic first integrals of Nosé-Hoover Equation (4).

Corollary 2. The following statements hold for Nosé-Hoover Equation (4):

(1) If $\alpha=0$, (4) has two functionally independent first integrals.

(2) If $\alpha \in\{1,1 / 2,-1 / 2\}$, (4) has at most one meromorphic first integrals.

(3) If $\alpha \notin\{0,1,1 / 2,-1 / 2\}$, (4) has no any meromorphic first integrals.

\subsection{Chaotic Systems}

Since the first chaotic Lorenz equations were derived, the chaos theory on dynamical systems has been widely studied and applied in a variety of fields such as physics, economics, secure communications, computer science, and so on. Roughly speaking, chaotic systems are dynamical systems which are highly sensitive to initial conditions. In this subsection, we try to investigate the non-integrability of the Rikitake-like system and the Lü system, both of which have been studied and, with some particular parameters, they can develop the chaotic phenomena $[29,30]$. 
Firstly, we consider the Rikitake-like system which reads:

$$
\left\{\begin{array}{l}
\dot{x}=-k x+y z \\
\dot{y}=-k y+(z-a) x \\
\dot{z}=z-x y
\end{array}\right.
$$

where the parameters $k$ and $a$ are positive. This system was proposed by Wu and Wang [29] in order to study the fractional-order chaotic and projective synchronization. The Rikitake-like system (10) exhibits a chaotic attractor for $k=2$ and $a=5$.

Obviously, system (10) has a phase curve $\Gamma_{2}=\left\{\left(0,0, e^{t}\right) \mid t \in \mathbb{C}\right\}$ and the norm variational equation along $\Gamma_{2}$ has the form:

$$
\left(\begin{array}{c}
\dot{\xi} \\
\dot{\eta}
\end{array}\right)=\left(\begin{array}{cc}
-k & e^{t} \\
e^{t}-a & -k
\end{array}\right)\left(\begin{array}{l}
\xi \\
\eta
\end{array}\right),
$$

which can be rewritten as:

$$
\ddot{\xi}=(1-2 k) \dot{\xi}+\left(e^{2 t}-a e^{t}+k-k^{2}\right) \xi
$$

We make the time scale transformation $\tau=e^{t}$ to transform this equation into an equation with rational coefficients, and so (12) is converted into:

$$
\xi^{\prime \prime}=-\frac{2 k}{\tau} \xi^{\prime}+\frac{k-k^{2}-a \tau+\tau^{2}}{\tau^{2}},
$$

where the prime is the derivation with respect to $\tau \in \mathbb{C}$. By Theorem A3, the above transformations do not change the identity component of the differential Galois group of (11). Similarly, making the change of dependent variable $\xi(\tau)=\chi(\tau) / \tau^{k}$ to eliminate the first order term, we obtain:

$$
\chi^{\prime \prime}=r \chi, \quad r(\tau)=\frac{\tau-a}{\tau}
$$

Then, introducing the new independent variable $z=2 \tau$, we transform (13) into the Whittaker equation:

$$
\frac{d^{2} \chi}{d z^{2}}-\left(\frac{1}{4}-\frac{a}{2 z}\right) \chi=0
$$

Set $\kappa=a / 2, \mu=1 / 2$. Then,

$$
\begin{aligned}
\kappa-\mu-\frac{1}{2} & =\frac{a}{2}-1, \\
\kappa+\mu-\frac{1}{2} & =\frac{a}{2} \\
-\kappa-\mu-\frac{1}{2} & =-\frac{a}{2}-1, \\
-\kappa+\mu-\frac{1}{2} & =-\frac{a}{2} .
\end{aligned}
$$

Then, due to Theorem A5, the identity component of (14) is solvable if and only if $a$ is an even integer.

In addition, the only pole of (14) is at zero and the corresponding order is 1 , and the order of $r$ at $\infty$ is 0 . It follows from Theorem A2 that case 3 cannot hold, which implies the differential Galois group of (14) is not finite. Then, as the differential Galois group of (14) can be viewed as a normal subgroup of the differential Galois group of (11), we obtain that the differential Galois group of (11) is not finite. By Corollary 1, we trivially have the following result: 
Theorem 4. The Rikitake-like system (10) is not meromorphically completely integrable. Moreover, assuming that the positive number $a$ is not an even integrer, then system (10) does not admit any meromorphic first integral in a neighborhood of $\Gamma_{2}$.

Now, we turn to consider the Lü system,

$$
\left\{\begin{array}{l}
\dot{x}=a(y-x), \\
\dot{y}=c y-x z \\
\dot{z}=x y-b z
\end{array}\right.
$$

which was first proposed in [30] and connected the Lorenz system with the Chen system. It is well known that (15) admits a chaotic attractor for $a=36, b=3$ and $c=20$. So far there have been a large number of related studies on the Lü system such as dynamical behaviors, chaotic control, chaos synchronization (see [38-40] and the references therein).

However, there are few results considering the integrability of (15). Llibre et al. [38,39] consider the Darboux first integrals and polynomial first integrals. Here, we investigate the first integrals of the Lü system in the category of meromorphic functions.

When $a$ vanishes, the Lü system has two functionally independent first integrals and it is completely integral (see [38]). Thus, in what follows, we assume $a \neq 0$. We also assume $b \neq 0$ to make sure that the particular solution $(x, y, z)=\left(0,0, e^{-b t}\right)$ is not a equilibrium.

Let $\Gamma_{3}$ be the associated phase curve. The norm variational equations along $\Gamma_{3}$ are:

$$
\left(\begin{array}{c}
\dot{\xi} \\
\dot{\eta}
\end{array}\right)=\left(\begin{array}{cc}
-a & a \\
-e^{-b t} & c
\end{array}\right)\left(\begin{array}{l}
\xi \\
\eta
\end{array}\right) .
$$

Then we transform (16) into a second order differential equation which gives:

$$
\ddot{\xi}+(a-c) \dot{\xi}-a\left(c-e^{-b t}\right) \xi=0 .
$$

Making the variable changes $\tau=e^{-b t}$, and $\xi(\tau)=\chi(\tau) \tau^{\frac{a-b-c}{2 b}}$, we obtain the following equivalent equations with rational coefficients:

$$
\chi^{\prime \prime}=r(\tau) \chi, r(\tau)=\left(-\frac{a}{b^{2} \tau}+\frac{(a+c)^{2}-b^{2}}{4 b^{2} \tau^{2}}\right),
$$

where the prime denotes the derivative with respect to $\tau$. Under a change of the independent variable $z=2 \sqrt{a \tau} / b$, then (17) becomes:

$$
z^{2} \frac{d^{2} \chi}{d z^{2}}-z \frac{d \chi}{d z}+\left(z^{2}-\frac{(a+c)^{2}-b^{2}}{b^{2}}\right) \xi=0
$$

Further, making the variable changes $\chi=\sqrt{z} \tilde{\chi}(z)$, we transform (18) into the Bessel equation in the reduced form:

$$
\frac{d^{2} \tilde{\chi}}{d z^{2}}=\left(\frac{4 n^{2}-1}{4 x^{2}}-1\right) \tilde{\chi}
$$

where $n=(a+c) / b$. Since (19) has two singular points 0 of order 1 and $\infty$ of order 2 . It follows that (19) could not fall in case 3. Hence, the differential Galois group of (19) is not finite. Further, thanks to Theorem A6, the identity component of (19) is solvable if and only if $2(a+c) / b$ is an odd integer.

By Corollary 1, we can trivially obtain the following non-integrable results. 
Theorem 5. Suppose $a b \neq 0$. Then the Lü system (15) is not meromorphically integrable. Moreover, if $2\left|\frac{a+c}{b}\right|$ is not an odd number, then system (15) does not possess any meromorphic first integral in a neighborhood of $\Gamma_{3}$.

Remark 2. When $a=36, b=3$ and $c=20$, it is well known that system (15) has a strange attractor. Note that in this case, $2\left|\frac{a+c}{b}\right|=\frac{112}{3}$ is not an odd number; using Theorem 5 we conclude that system (15) admits no meromorphic first integrals. This fact can be seen as a new evidence of the connection between the chaos and the non-integrability.

Remark 3. If $2\left|\frac{a+c}{b}\right|$ is an odd number, then system (15) may have a meromorphic first integral. For example, for $a=\frac{1}{2}, b=1, c=-1$, system (15) admits a meromorphic first integral:

$$
\Phi(x)=\frac{2\left(x^{2}-z\right)^{2}}{y^{2}-1+2 x^{2} z} .
$$

Further, by Theorem 5, in this case, (15) has only one meromorphic first integral in a neighborhood of $\Gamma_{4}$ in the sense of functional independence.

\subsection{Rucklidge System}

Consider the following differential equations:

$$
\left\{\begin{array}{l}
\dot{x}=-a x+b y-y z \\
\dot{y}=x \\
\dot{z}=-z+y^{2}
\end{array}\right.
$$

which are introduced to describe two-dimensional convection in a horizontal layer of Boussinesq fluid with lateral constants [31]. It is also one of three-dimensional chaotic systems, which is not topologically equivalent to the Lorenz system. The chaotic behavior occurs when the parameter values are taken as $a=2$ and $b=6.7$ with the initial condition $\left(x_{0}, y_{0}, z_{0}\right)=(1,0,4.5)$. The dynamical features of the Rucklidge system have been investigated in the last decades. Hopf bifurcations and small amplitude limit cycles of (20) were considered in [41]. The control of chaos of the Rucklidge system was investigated in [42]. In view of the integrability, Lima et al. in [43] proved that the Rucklidge model has no analytic first integrals. The aim of this subsection is to consider the meromophic integrability, and the main result related to the integrability problem of (20) is summarized in the next result.

Theorem 6. The Rucklidge model is not meromorphic completely integrable. Moreover, it has no any meromorphic first integral in the neighbourhood of $\Gamma_{4}$ if one of the following conditions holds:

(1) $b=0,2 a \notin \mathbb{Z}$

(2) $b \neq 0,4\left(a^{2}+4 b\right) \neq(2 n-1)^{2}$ for all $n \in \mathbb{N}$.

Here, $\Gamma_{4}$ is the phase curve with the particular non-equilibrium solution $(x, y, z)=\left(0,0, e^{-t}\right)$. The linearized system of Equation (20) of variations in normal directions, i.e., normal variational equations, is derived as follows:

$$
\left(\begin{array}{l}
\dot{\zeta} \\
\dot{\eta}
\end{array}\right)=\left(\begin{array}{cc}
-a & b-e^{-t} \\
1 & 0
\end{array}\right)\left(\begin{array}{l}
\xi \\
\eta
\end{array}\right),
$$

or equivalently,

$$
\ddot{\xi}=\frac{e^{-t}-a b+a e^{-t}}{b-e^{-t}} \dot{\xi}+\frac{b^{2}-2 b e^{-t}+e^{-2 t}+a e^{-t}}{b-e^{-t}} \xi .
$$


In an argument similar to the above models, we successively make the variable changes $\tau=e^{-t}$, $\xi=\chi \sqrt{\tau^{a-1}(\tau-b)}$ and transform (22) into the confluent Heun equation in the reduced form:

$$
\chi^{\prime \prime}=r \chi, r=\frac{\left(a^{2} b^{2}+4 b^{3}-b^{2}\right)+\left(2 a b-2 a^{2} b-12 b^{2}+4 b\right) \tau+\left(a^{2}-2 a+12 b\right) \tau^{2}-4 \tau^{3}}{4 \tau^{2}(b-\tau)^{2}},
$$

where the prime denotes the derivative with respect to $\tau$. In what follows, we use the Kovacic algorithm to study the differential Galois group of (23).

No matter the values of the parameters $a$ and $b$, the order of $\infty$ is one. It follows from Theorem A2 that cases 1, 3 do not hold. By Corollary 1, the Rucklidge system (20) is not meromorphically completely integrable. To complete Theorem 6, we apply Kovacic algorithm of case 2 to (23), and show that case 2 cannot hold if $2 a \notin \mathbb{Z}$ when $b=0$ or $a^{2}+4 b \neq \frac{(2 n-1)^{2}}{4}$ for any $n \in \mathbb{N}$ when $b \neq 0$.

When $b=0$, (23) becomes:

$$
\chi^{\prime \prime}=r \chi, r=\left(\frac{a(a-2)}{4 \tau^{2}}-\frac{1}{\tau}\right)
$$

It has only one pole at 0 . Obviously, $a(a-2) \neq 0$ as $2 a \notin \mathbb{Z}$. Let,

$$
e_{0} \in\{2 \pm 2|a-1|\} \cap \mathbb{Z}, \quad e_{\infty} \in\{1\} .
$$

Then $\left(e_{\infty}-e_{0}\right) / 2$ must be a non-negative integer to assure case 2 holds. Clearly, it is impossible for $2 a \notin \mathbb{Z}$.

When $b \neq 0$, (23) becomes,

$$
\chi^{\prime \prime}=r \chi, r=\frac{a^{2}+4 b-1}{4 \tau^{2}}+\frac{3}{4(\tau-b)^{2}}+\frac{a-2 b+1}{2 b \tau}-\frac{1+a}{2 b(\tau-b)} .
$$

If $a^{2}+4 b=1$, then the poles of $r$ are $\mathcal{P}=\{0, b, \infty\}$, and the order at $\tau=0, b$ and $\infty$ are 1,2 and 1 . Let,

$$
E_{0}=\{4\}, E_{b}=\{2,-2,6\}, E_{\infty}=\{1\}
$$

It is easy to check that $d=\frac{1}{2}\left(e_{\infty}-e_{0}-e_{b}\right)$ is not a non-negative integer for all the families $\left(e_{c}\right)_{c \in \mathcal{P}}$. Thus case 2 should be excluded if $a^{2}+4 b \neq 1$. Proceeding as above, let

$$
E_{0}=\left\{2,2+2 \sqrt{a^{2}+4 b}, 2-2 \sqrt{a^{2}+4 b}\right\} \cap \mathbb{Z}, E_{b}=\{2,-2,6\}, E_{\infty}=\{1\} .
$$

To make sure that there exist families $\left(e_{c}\right)_{c \in \mathcal{P}}$ such that $d=\frac{1}{2}\left(e_{\infty}-e_{0}-e_{b}\right) \in \mathbb{N} \cup\{0\}$, we know that $4\left(a^{2}+4 b\right)$ should be the square of an odd number, which contradicts the assumption.

Acknowledgments: We are extremely grateful to the reviewers for careful reading of the manuscript and valuable comments. This work was funded by The NSF (No. 11371166, 11301210) of China, China Automobile Industry Innovation and Development Joint Fund (No. U1664257), NSF (20140520053JH) of Jilin, China and PSF (2014M560038) of China, ESF (JJKH20170776KJ) of Jilin, China.

Author Contributions: Kaiyin Huang, Shaoyun Shi and Wenlei Li collectively realized theoretical analyses; Kaiyin Huang wrote the paper. All authors have read and approved the final manuscript.

Conflicts of Interest: The authors declare no conflict of interest.

\section{Appendix A}

Let us consider the following differential equation with rational coefficients:

$$
\frac{d^{2} \xi}{d z^{2}}+p(z) \frac{d \xi}{d z}+q(z) \xi(z)=0, p(z), q(z) \in \mathbb{C}(z) .
$$


If we know a non-zero solution $\xi_{1}(z)$ of (A1), then another solution $\xi_{2}(z)$, linearly independent from $\xi_{1}(z)$, is given by:

$$
\xi_{2}(z)=\xi_{1} \int \frac{1}{\xi_{1}^{2}} \exp \left[-\int p\right]
$$

Making the change of dependent variable,

$$
\xi(z)=\chi(z) \exp \left(-\int \frac{1}{2} p\right)
$$

the Equation (A1) is transformed to the reduced form:

$$
\frac{d^{2} \chi}{d z^{2}}=r(z) \chi, \quad r(z)=\frac{1}{4} p(z)^{2}+\frac{1}{2} \frac{d p}{d z}-q(z) \in \mathbb{C}(z) .
$$

It should be pointed out that the above transformation does not affect the Liouvillian solvability of (A1), and so the identity component $G^{0}$ of (A3) is solvable if and only if that of (A1) is solvable. Moreover, by the Galois correspondence, we see that the differential Galois group of (A3) can be viewed as a normal subgroup of the differential Galois group of (A1) (for more details see Theorem 2.5 in [11]). Therefore, if the identity component $G^{0}$ of (A3) is not finite (or abelian), then the identity component $G^{0}$ of (A1) is also not finite (or abelian). Let $S L(2, \mathbb{C})$ be the set of $2 \times 2$ matrices with determinant one. Then, the differential Galois group $G$ of (A3) is an algebraic group contained in $S L(2, \mathbb{C})$.

The following result gives a classification of the differential Galois group $G$ of (A3), for the proof see Lemma 1.4 in [34].

Theorem A1. There are four cases that can occur:

Case1. G is conjugate to a triangular group. Then $(A 3)$ has a solution of the form $e^{\int \omega}$ with $\omega \in \mathbb{C}(x)$.

Case2. G is not of case 1, but is conjugate to a subgroup of,

$$
D=\left\{\left(\begin{array}{cc}
c & 0 \\
0 & c^{-1}
\end{array}\right) \mid c \in \mathbb{C}, c \neq 0\right\} \cup\left\{\left(\begin{array}{cc}
0 & c \\
-c^{-1} & 0
\end{array}\right) \mid c \in \mathbb{C}, c \neq 0\right\} .
$$

Then $(A 3)$ has a solution of the form $e^{\int \omega}$ with $\omega$ algebraic over $\mathbb{C}(x)$ of degree 2 .

Case3. $G$ is not of case 1 and case 2, but is a finite group. Then all solutions of $(A 3)$ are algebraic over $\mathbb{C}(x)$.

Case4. $G=S L(2, \mathbb{C})$. Then $(A 3)$ is not integrable in Liouville sense.

Kovacic in [34] also gave some necessary conditions that should be satisfied by $r(z)$ when cases 1 , 2,3 hold. Before their formulation, it is useful to fix some terminology. Let $r(z)$ be the ratio $s(z) / t(z)$ of two relative polynomials $s(z), t(z) \in \mathbb{C}[z]$. The order of a pole is defined as the multiplicity of the corresponding root of $t(z)$. The order of $r$ at $\infty$ is $\operatorname{deg} t(z)-\operatorname{deg} s(z)$.

Theorem A2. The following conditions are necessary for the respective cases of Theorem A1 to hold.

Case1. Every pole of $r$ must have even order or else have order 1 . The order of $r$ at $\infty$ must be even or else be great than 2.

Case2. $\quad r$ must have at least one pole such that either has odd order greater than 2 or else has order 2.

Case3. The order of a pole of $r$ cannot exceed 2 and the order of $r$ at $\infty$ must be at least 2 . If the partial fraction expansion of $r$ is,

$$
r=\sum_{i} \frac{\alpha_{i}}{\left(x-\beta_{i}\right)^{2}}+\sum_{i} \frac{\gamma_{j}}{x-d_{i}}
$$

then,

$$
\sqrt{1+4 \alpha_{i}} \in \mathbb{Q}, \quad \sum_{j} \gamma_{j}=0, \quad \sqrt{\sum_{i} \alpha_{i}+\sum_{i} \gamma_{j} d_{i}} \in \mathbb{Q}
$$


Let us make a remark that the identity components $G^{0}$ in cases $1-3$ are solvable, but in case 4 it is not. Due to Corollary 1, if $G$ falls into case 4, then system (1) has no any meromorphic first integrals. Moreover, from the proof of Theorem A1 by Kovacic, we also see that the necessary condition for case 3 is based on the assumption that $G$ is a finite group and admits an algebraic solution, which has nothing to do with the assumption that cases 1 and 2 do not hold. Hence, if the necessary condition for case 3 is evaluated to be false, then $G$ is not a finite group and by Corollary 1 system (1) is not completely integrable with meromorphic first integrals.

Before formulating Kovacic's algorithm, let us remark that if the coefficients of the normal variational equations are not rational functions but are meromorphic with respect to the independent variational, then we cannot use Kovacic's Algorithm directly but make proper change of variables to do so (see Theorem 2.5 in [11]):

Theorem A3. Let $X$ be a (connected) Riemann surface. Let $f: X^{\prime} \rightarrow X$ be a finite ramified covering of $X$ by a Riemann surface $X^{\prime}$. Let $\nabla$ be a meromorphic connection on $X$, and set $\nabla^{\prime}=f^{*} \nabla$. Then, we have a natural injective homomorphism $\operatorname{Gal}\left(\nabla^{\prime}\right) \rightarrow \operatorname{Gal}(\nabla)$ of differential Galois groups which induces an isomorphism between their Lie algebras(and identity component part).

In other words, if the original differential equation over the Riemann surface $X^{\prime}$ is transformed by a change of the independent variable in a differential equation over the Riemann surface $X$, then both equations have the same identity component of the differential Galois group in the sense of isomorphism.

In what follows, we formulate the complete Kovacic's algorithm, which can help us recognize which cases the differential Galois group of (A3) belongs to.

\section{Appendix A1. Kovacic's Algorithm of Case 1}

- Step 1. For each $c \in \mathcal{P}$ we define $[\sqrt{r}]_{c}, \alpha_{c}^{ \pm}$as follows:

(a) If $c$ is a pole of order 1 , then $[\sqrt{r}]_{c}=0, \alpha_{c}^{+}=\alpha_{c}^{-}=1$.

(b) If $c$ is a pole of order 2, then $[\sqrt{r}]_{c}=0, \alpha_{c}^{ \pm}=\frac{1}{2} \pm \frac{1}{2} \sqrt{1+4 b}$, where $b$ is the coefficient of $1 /(x-c)^{2}$ in the partial fraction expansion for $r$.

(c) If $c$ is a pole of order $2 v \geq 4$, then $[\sqrt{r}]_{c}=\frac{a}{(x-c)^{v}}+\cdots+\frac{d}{(x-c)^{2}}$ of negative order part of the Laurent series expansion of $\sqrt{r}$ at $c, \alpha_{c}^{ \pm}=\frac{1}{2}\left( \pm \frac{b}{a}+v\right)$, where $b$ is the coefficient of $1 /(x-c)^{v+1}$ in $r$ minus the coefficient of $1 /(x-c)^{v+1}$ in $[\sqrt{r}]_{c}^{2}$.

(d) If the order of $r$ at $\infty$ is $>2$, then $[\sqrt{r}]_{\infty}=0, \alpha_{\infty}^{+}=0, \alpha_{\infty}^{-}=1$.

(e) If the order of $r$ at $\infty$ is 2, then $[\sqrt{r}]_{\infty}=0, \alpha_{\infty}^{ \pm}=\frac{1}{2} \pm \frac{1}{2} \sqrt{1+4 b}$, where $b$ is the coefficient of $1 / x^{2}$ in the Laurent series expansion of $r$ at $\infty$.

(f) If $\infty$ is a pole of order $-2 v \leq 0$, then $[\sqrt{r}]_{\infty}=a x^{v}+\cdots+d$ of the positive order part of the Laurent series expansion of $\sqrt{r}$ at $\infty, \alpha_{\infty}^{ \pm}=\frac{1}{2}\left( \pm \frac{b}{a}-v\right)$, where $b$ is the coefficient of $x^{v-1}$ in $r$ minus the coefficient of $x^{v-1}$ in $[\sqrt{r}]_{\infty}^{2}$.

- Step 2. Let $d=\sum_{c \in \mathcal{P}} t(c) \alpha_{c}^{s(c)}$, where $s(c) \in\{+,-\}$ for any $c \in \mathcal{P}, t(\infty)=+1$ and $t(c)=-1$ for any $c \in \mathcal{P} \backslash\{\infty\}$. If $d$ is a non-negative integer, then let $w=\sum_{c \in \mathcal{P}}\left(s(c)[\sqrt{r}]_{c}+\frac{\alpha_{c}^{s(c)}}{x-c}\right)+[\sqrt{r}]_{\infty} s(\infty)$, otherwise, the family is discarded. If no families remain under consideration, case 1 of Theorem A1 cannot happen.

- Step 3. For each family retained from step 2, we search for a monic polynomial $P$ of degree $d$ such that the equation $P^{\prime \prime}+2 w P^{\prime}+\left(w^{\prime}+w^{2}-r\right) P=0$ holds. If such a polynomial exists, then $\xi=P e^{\int w}$ is a solution of $\xi^{\prime \prime}=r \xi$. If no such polynomial is found for any family retained from Step 2, case 1 of Theorem A1 cannot happen. 
Appendix A2. Kovacic's Algorithm of Case 2

- Step 1. For each $c \in \mathcal{P}$ we define $E_{c}$ as follows:

(a) If $c$ is a pole of order 1 , then $E_{c}=\{4\}$.

(b) If $c$ is a pole of order 2, then $E_{c}=\{2+k \sqrt{1+4 b}, k=0, \pm 2\} \cap \mathbb{Z}$, where $b$ refers to coefficients of $1 /(x-c)^{2}$ in the partial fraction expansion for $r$.

(c) If $c$ is a pole of order $v>2$, then $E_{c}=\{v\}$.

(d) If the order of $r$ at $\infty$ is $>2$, then $E_{\infty}=\{0,2,4\}$.

(e) If the order of $r$ at $\infty$ is 2 , then $E_{\infty}=\{2+k \sqrt{1+4 b}, k=0, \pm 2\} \cap \mathbb{Z}$, where $b$ refers to coefficients of $1 / x^{2}$ in the Laurent series expansion of $r$ at $\infty$.

(f) If $\infty$ is a pole of order $v<2$, then $E_{\infty}=\{v\}$.

- Step 2. Let $d=\frac{1}{2} \sum_{c \in \mathcal{P}} t(c) e_{c}$, where $e_{c} \in E_{c}$ for any $c \in \mathcal{P}, t(\infty)=+1$ and $t(c)=-1$ for any $c \in \mathcal{P} \backslash\{\infty\}$. If $d$ is a non-negative integer, then let $\theta=\frac{1}{2} \sum_{c \in \mathcal{P}} \frac{e_{c}}{x-c}$, otherwise, the family is discarded. If no families remain under consideration, case 2 of Theorem A1 cannot happen.

- Step 3. For each family retained from step 2, we search for a monic polynomial $P$ of degree $d$ such that the equation $P^{\prime \prime \prime}+3 \theta P^{\prime \prime}+\left(3 \theta^{2}+3 \theta^{\prime}-4 r\right) P^{\prime}+\left(\theta^{\prime \prime}+3 \theta \theta^{\prime}+\theta^{3}-4 r \theta-2 r^{\prime}\right) P=0$ holds. If such a polynomial exists, let $\phi=\theta+\frac{P^{\prime}}{P}$ and let $w$ be a solution of the equation $w^{2}+\phi w+\left(\frac{1}{2} \phi^{\prime}+\right.$ $\left.\frac{1}{2} \phi^{2}-r\right)=0$, then $\xi=e^{\int w}$ is a solution of $\xi^{\prime \prime}=r \xi$. If no such polynomial is found for any family retained from Step 2, case 2 of Theorem A1 cannot happen.

Appendix A3. Kovacic's Algorithm of Case 3

- Step 1. For each $c \in \mathcal{P}$ we define $E_{c}$ as follows:

(a) If $c$ is a pole of order 1 , then $E_{c}=\{12\}$.

(b) If $c$ is a pole of order 2, then $E_{c}=\left\{6+\frac{12 k}{n} \sqrt{1+4 b}, k=0, \pm 1, \pm 2, \cdots, \pm \frac{n}{2}\right\} \cap \mathbb{Z}$, where $b$ is a coefficient of $1 /(x-c)^{2}$ in the partial fraction expansion for $r$. Here and below in this case $n \in\{4,6,12\}$.

(c) $E_{\infty}=\left\{6+\frac{12 k}{n} \sqrt{1+4 b}, k=0, \pm 1, \pm 2, \cdots, \pm \frac{n}{2}\right\} \cap \mathbb{Z}$, where $b$ refers to coefficients of $1 / x^{2}$ in the Laurent series expansion of $r$ at $\infty$.

- Step 2. Let $d=\frac{n}{12} \sum_{c \in \mathcal{P}} t(c) e_{c}$, where $e_{c} \in E_{c}$ for any $c \in \mathcal{P}, t(\infty)=+1$ and $t(c)=-1$ for any $c \in \mathcal{P} \backslash\{\infty\}$. If $d$ is a non-negative integer, then let $\theta=\frac{n}{12} \sum_{c \in \mathcal{P}} \frac{e_{c}}{x-c}, S=\prod_{c \in \mathcal{P} \backslash\{\infty\}}(x-c)$, otherwise, the family is discarded. If no families remain under consideration, case 3 of Theorem A1 cannot happen.

- Step 3. For each family retained from step 2, we search for a monic polynomial $P$ of degree $d$ such that the recursive equations:

$$
\begin{gathered}
P_{n}=-P, \\
P_{i-1}=-S P_{i}+\left((n-i) S^{\prime}-S \theta\right) P_{i}-(n-i)(i+1) S^{2} r P_{i+1} ;(i=n, n-1, \cdots, 0),
\end{gathered}
$$

with $P_{-1} \equiv 0$ hold. If such a polynomial exists, let $w$ be a solution of the equation $\sum_{i=0}^{n} \frac{S^{i} P_{i}}{(n-i) !} w^{i}=0$, then $\xi=e^{\int w}$ is a solution of $\xi^{\prime \prime}=r \xi$. If no such polynomial is found for any family retained from Step 2, case 3 of Theorem A1 cannot happen.

\section{Appendix B}

In this section, we present some known facts about linear differential equations of special forms. First, we consider a class of two order linear differential equations, which are given by:

$$
\frac{d^{2} \xi}{d z^{2}}-\left(a z^{2}+b z+c\right) \xi=0,
$$


where $a \neq 0, b, c$ are parameters. As an example to illustrate his algorithm, Kovacic gave necessary and sufficient conditions for which (A4) admits a Liouvillian solution.

Theorem A4. The identity component $G^{0}$ of $(A 4)$ is solvable if and only if $\left(4 a c-b^{2}\right) / 4 a^{2}$ is an odd integer.

Remark A1. A special case of this example is the Weber equation:

$$
\frac{d^{2} \xi}{d z^{2}}-\left(\frac{1}{4} z^{2}-\frac{1}{2}+n\right) \xi=0, \quad n \in \mathbb{C},
$$

where $a=\frac{1}{2}$ and $b=-\frac{1}{2}+n$. Hence we see that the Weber equation is solvable if and only if $n$ is an integer.

Next, we consider the Whittaker equation:

$$
\frac{d^{2} \xi}{d z^{2}}-\left(\frac{1}{4}-\frac{\kappa}{z}+\frac{4 \mu^{2}-1}{4 z^{2}}\right) \xi=0,
$$

where $\kappa, \mu$ are parameters. Using Stokes multipliers, Morales-Ramis in [11] studied the differential Galois group of (A5). The following result presents necessary and sufficient conditions for solvability of the identity component of the differential Galois group of (A5).

Theorem A5. The identity component $G^{0}$ of (A5) is solvable if and only if at least one of the four numbers $\kappa-\mu-\frac{1}{2}, \kappa+\mu-\frac{1}{2},-\kappa-\mu-\frac{1}{2}$ and $-\kappa+\mu-\frac{1}{2}$ is a positive integer.

Finally, we consider the Bessel equation,

$$
z^{2} \frac{d^{2} \xi}{d z^{2}}+z \frac{d \xi}{d z}+\left(z^{2}-n^{2}\right) \xi=0
$$

where $n$ is the parameter. It admits two singular points 0 and $\infty$. Making the variable changes $\xi(z)=\tilde{\xi} / \sqrt{z}$, we can transform (A6) into the Bessel equation in the reduced form:

$$
\frac{d^{2} \tilde{\xi}}{d z^{2}}=\left(\frac{4 n^{2}-1}{4 z^{2}}-1\right) \tilde{\xi} .
$$

For the solvability of identity component of the differential Galois group associated with (A7), we have the following theorem due to Morales-Ruiz [11].

Theorem A6. The identity component $G^{0}$ of $(A 7)$ is solvable if and only if $n+1 / 2$ belongs to $\mathbb{Z}$.

\section{References}

1. Leonov, G.A.; Alexeeva, T.A.; Kuznetsov, N.V. Analytic Exact Upper Bound for the Lyapunov Dimension of the Shimizu-Morioka System. Entropy 2015, 17, 5101-5116.

2. Wang, Z.; Ma, J.; Chen, Z.Q.; Zhang, Q. A New Chaotic System with Positive Topological Entropy. Entropy 2015, 17, 5561-5579.

3. Wang, S.; Wang, X.; Zhou, Y. A memristor-based complex Lorenz system and its modified projective synchronization. Entropy 2015, 17, 7628-7644.

4. Bolsinov, A.V.; Taimanov, I.A. Integrable geodesic flows with positive topological entropy. Invent. Math. 2000, 140, 639-650.

5. Yagasaki, K. Galoisian obstructions to integrability and Melnikov criteria for chaos in two-degree-of-freedom Hamiltonian systems with saddle centres. Nonlinearity 2003, 16, 2003-2012.

6. Goriely, A. Integrability and nonintegrability of dynamical systems. World Sci. 2002, 122, 259-275.

7. Bountis, T.C.; Ramani, A.; Grammaticos, B.; Dorizzi, B. On the complete and partial integrability of non-Hamiltonian systems. Phys. A 1984, 128, 268-288. 
8. Lakshmanan, M.; Sahadevan, R. Painlevé analysis, Lie symmetries, and integrability of coupled nonlinear oscillators of polynomial type. Phys. Rep. 1993, 224, 1-93, doi:10.1016/0370-1573(93)90081-N.

9. Christopher, C.; Llibre, J.; Pereira, J.V. Multiplicity of invariant algebraic curves in polynomial vector fields. Pac. J. Math. 2007, 229, 63-117.

10. Ziglin, S.L. Branching of solutions and nonexistence of first integrals in Hamiltonian mechanics I, II. Funct. Anal. Appl. 1982, 16, 181-189.

11. Morales-Ruiz, J.J. Differential Galois Theory and Non-Integrability of Hamiltonian Systems; Birkhäuser: Basel, Switzerland, 1999.

12. Morales-Ruiz, J.J.; Simó, C. Picard-Vessiot Theory and Ziglin's Theorem. J. Differ. Equ. 1994, 107, $140-162$.

13. Morales-Ruiz, J.J.; Ramis, J.P. Galoisian obstructions to integrability of Hamiltonian systems. I. Methods Appl. Anal. 2001, 8, 33-95.

14. Morales-Ruiz, J.J.; Ramis, J.P. Galoisian obstructions to integrability of Hamiltonian systems. II. Methods Appl. Anal. 2001, 8, 97-111.

15. Baider, A.; Churchill, R.C.; Rod, D.L.; Singer, M.F. On the infinitesimal geometry of integrable systems. Mech. Day 1996, 7, 5-56.

16. Churchill, R.C.; Rod, D.L.; Singer, M.F. Group-theoretic obstructions to integrability. Ergod. Theory Dyn. Syst. $1995,15,15-48$.

17. Maciejewski, A.J.; Przybylska, M.; Simpson, L.; Szumiski, W. Non-integrability of the dumbbell and point mass problem. Celest. Mech. Dyn. Astronom. 2013, 117, 315-330.

18. Stachowiak, T.; Szumiski, W. Non-integrability of restricted double pendula. Phys. Lett. A 2015, 379, 3017-3024.

19. Maciejewski, A.J.; Przybylska, M.; Weil, J. Non-integrability of the generalized spring-pendulum problem. J. Phys. A 2004, 37, 2579-2597.

20. Przybylska, M.; Szumiski, W. Non-integrability of flail triple pendulum. Chaos Solitons Fractals 2013, 53, 60-74.

21. Li, W.L.; Shi, S.Y. Galoisian obstruction to the integrability of general dynamical systems. J. Differ. Equ. 2012, 252, 5518-5534.

22. Li, W.L.; Shi, S.Y. Corrigendum to "Galoisian obstruction to the integrability of general dynamical systems". J. Differ. Equ. 2017, 262, 1253-1256.

23. Ayoul, M.; Zung, N.T. Galoisian obstructions to non-Hamiltonian integrability. C. R. Math. Acad. Sci. 2010, $348,1323-1326$.

24. Przybylska, M. Differential Galois obstructions for integrability of homogeneous Newton equations. J. Math. Phys. 2008, 49, 022701.

25. Maciejewski, A.J.; Przybylska, M. Non-integrability of ABC flow. Phys. Lett. A 2002, 303, 265-272.

26. Ziglin, S.L. An analytic proof of the nonintegrability of the ABC-flow for A = B = C. Funct. Anal. Appl. 2003, 37, 225-227.

27. Nishiyama, T. Meromorphic non-integrability of a steady Stokes flow inside a sphere. Ergod. Theory Dyn. Syst. 2014, 34, 616-627.

28. Hoover, W.G. Canonical dynamics: Equilibrium phase-space distributions. Phys. Rev. A 1985, 31, $1695-1697$.

29. Wu, X.; Wang, H. A new chaotic system with fractional order and its projective synchronization. Nonlinear Dyn. 2010, 61, 407-417.

30. Lü, J.H.; Chen, G.R. A new chaotic attractor coined. Int. J. Bifurc. Chaos Appl. Sci. Eng. 2002, 12, $659-661$.

31. Rucklidge, A.M. Chaos in models of double convection. J. Fluid Mech. 1992, 237, 209-229.

32. Llibre, J.; Valls, C.; Zhang, X. The completely integrable differential systems are essentially linear differential systems. J. Nonlinear Sci. 2015, 25, 815-826.

33. Van der Put, M.; Singer, M.F. Galois Theory of Linear Differential Equations; Springer: Berlin, Germany, 2012.

34. Kovacic, J. An algorithm for solving second order linear homogeneous differential equations. J. Symb. Comput. 1986, 2, 3-43.

35. Hamilton, I.; Brumer, P. Regular-to-irregular transition in conservative Hamiltonian systems: Critical energies and local entropies. Phys. Rev. A 1981, 23, 1941-1951.

36. Legoll, F.; Luskin, M.; Moeckel, R. Non-ergodicity of the Nosé-Hoover thermostatted harmonic oscillator. Arch. Ration Mech. Anal. 2007, 184, 449-463.

37. Mahdi, A.; Valls, C. Integrability of the Nosé-Hoover equation. J. Geom. Phys. 2011, 61, 1348-1352. 
38. Llibre, J.; Valls, C. Polynomial first integrals for the Chen and Lü systems. Int. J. Bifur. Chaos Appl. Sci. Eng. 2012, 22, 1250262.

39. Llibre, J.; Mahdi, A.; Valls, C. Darboux integrability of the Lü system. J. Geom. Phys. 2013, 63, 118-128.

40. Mello, L.F.; Coelho, S.F. Degenerate Hopf bifurcations in the Lü system. Phys. Lett. A 2009, 373, 1116-1120.

41. Dias, F.S.; Mello, L.F. Hopf bifurcations and small amplitude limit cycles in Rucklidge systems. Electron. J. Differ. Equ. 2013, 48, 1-9.

42. Kocamaz, U.; Uyaroğlu, Y. Controlling Rucklidge chaotic system with a single controller using linear feedback and passive control methods. Nonlinear Dyn. 2014, 75, 63-72.

43. Lima, M.F.S.; Llibre, J.; Valls, C. Integrability of the Rucklidge system. Nonlinear Dyn. 2014, 77, 1441-1453.

(C) 2017 by the authors. Licensee MDPI, Basel, Switzerland. This article is an open access article distributed under the terms and conditions of the Creative Commons Attribution (CC BY) license (http://creativecommons.org/licenses/by/4.0/). 